\title{
Synthesis and Characterization of Colloidal-Supported Metal Nanoparticles as Potential Intermediate Nanocatalysts
}

\author{
Kalyani Gude and Radha Narayanan* \\ Department of Chemistry, University of Rhode Island, Kingston, Rhode Island 02881
}

Received: January 4, 2010; Revised Manuscript Received: March 1, 2010

\begin{abstract}
We report the synthesis of a new type of intermediate nanocatalyst material that we term as colloidal-supported metal nanoparticles (CSMNs). The CSMNs that we have synthesized consist of palladium nanoparticles covalently attached to functionalized silica colloids. The synthesis process involves four steps, which include the synthesis of the silica colloids, synthesis of the palladium nanoparticles, functionalization of the silica colloids with 3-mercaptopropyl trimethoxysilane (MPTMS) or 3-aminopropyl triethoxysilane (APTES), and covalent attachment of the palladium nanoparticles onto the functionalized silica colloids. We have characterized the size of the silica colloids and palladium nanoparticles by using transmission electron microscopy (TEM). In addition, we have characterized the attachment of the palladium nanoparticles onto the two types of functionalized silica colloids by using TEM as well as energy dispersive spectroscopy (EDS). In the case of the CSMNs prepared by using the silica colloids functionalized with MPTMS, we observed that there is a significant amount of additional deposits between each of the functionalized silica colloids. This type of additional deposits is significantly diminished in the case of the CSMNs prepared by using silica colloids functionalized with APTES. We have conducted some initial studies to determine at what stage the additional deposits occur and discuss potential sources that give rise to this phenomenon. We have also conducted initial kinetics study and assessment of the stability of the CSMNs after the catalytic process.
\end{abstract}

\section{Introduction}

Metal nanoparticles are very attractive catalysts compared to bulk catalytic materials due to their high surface-to-volume ratio. Some types of traditional nanocatalysts include transition metal nanoparticles in colloidal suspension, ${ }^{1-8}$ those adsorbed onto bulk supports, ${ }^{9-14}$ and lithographically fabricated arrays of nanocatalysts. ${ }^{15-19}$ Transition metal nanoparticles in colloidal suspension have been synthesized by using a wide variety of reducing agents ${ }^{20}$ such as hydrogen, ${ }^{21-23}$ sodium borohydride, ${ }^{24}$ and ethanol. ${ }^{25-27}$ Many different types of stabilizers have been used as capping agents to stabilize the nanoparticles such as surfactants, ${ }^{28,29}$ polymers, ${ }^{29,30}$ dendrimers, ${ }^{28,30}$ as well as different types of ligands. ${ }^{28,29}$ In the case of metal nanoparticles adsorbed onto bulk supports, a wide variety of support materials have been used such as carbon, ${ }^{31,32}$ mesoporous silica, ${ }^{33,34}$ titania, ${ }^{35,36}$ alumina, ${ }^{36,37}$ zeolites, ${ }^{9,36}$ and resins. ${ }^{36,38}$ Arrays of metal nanocatalysts have been fabricated by using electron beam lithography ${ }^{17,19}$ as well as colloidal lithographic techniques. ${ }^{39}$

In this paper, we discuss the design of a new type of intermediate nanocatalyst that we term as colloidal-supported metal nanoparticles (CSMNs). Some potential advantages of CSMNs as intermediate nanocatalysts include the following: (1) being suspended in solution during liquid-phase catalytic reactions, (2) having high metal loadings on the silica colloid surface resulting in a high surface area, and (3) facile separation of the reaction mixture from the CSMNs. The CSMNs combine the advantages of heterogeneous catalysts in a near homogeneous format. These advantages make using CSMNs particularly attractive nanocatalysts for liquid-phase reactions compared to their colloidal counterparts and those adsorbed onto bulk supports. We have utilized a four-step process for synthesizing

* To whom correspondence should be addressed. E-mail: rnarayanan@ chm.uri.edu. Phone: 401-874-2298. Fax: 401-874-5072. the palladium nanoparticles attached to silica colloids. The four steps in this process include synthesizing the silica colloids, synthesizing the PVP-capped palladium nanoparticles, functionalizing the silica colloids, and attaching the PVP-capped palladium nanoparticles to the silica colloids. We have characterized the CSMNs by using TEM and EDS. In the case of the CSMNs prepared with silica colloids functionalized with 3-mercaptopropyl trimethoxysilane (MPTMS), there is a significant amount of additional deposits present between each of the silica colloids. This type of additional deposits is significantly diminished in the case of the CSMNs prepared with silica colloids functionalized with APTES. We have conducted some initial studies to determine at which stage the additional deposits are formed and discuss potential sources that give rise to this phenomenon.

\section{Experimental Section}

Synthesis of Silica Colloids. The silica colloids were synthesized by using the Stoeber synthesis method. ${ }^{40}$ First, 30 $\mathrm{mL}$ of ethanol and $2.4 \mathrm{~mL}$ of ammonium hydroxide were added to an Erlenmeyer flask and the solution was stirred for $5 \mathrm{~min}$. Then, $1.2 \mathrm{~mL}$ of tetraethylorthosilicate (TEOS) was added to the solution containing ethanol and ammonium hydroxide and this solution was stirred overnight. The solution was initially clear, after 15-20 min the solution starts to turn cloudy, and the final solution is very turbid and consists of a suspension of the silica colloids.

Synthesis of PVP-Stabilized Palladium Nanoparticles. The palladium nanoparticles were synthesized by using the ethanol reduction method similar to that described previously. ${ }^{25,41-43}$ The palladium precursor solution $\left(\mathrm{H}_{2} \mathrm{PdCl}_{4}\right)$ was prepared by adding $0.0887 \mathrm{~g}$ of $\mathrm{PdCl}_{2}$ and $6 \mathrm{~mL}$ of $0.2 \mathrm{M} \mathrm{HCl}$, then diluting to 250 $\mathrm{mL}$ with doubly distilled water. A solution containing $15 \mathrm{~mL}$ of $2 \mathrm{mM} \mathrm{H}_{2} \mathrm{PdCl}_{4}, 21 \mathrm{~mL}$ of doubly deionized water, $0.0667 \mathrm{~g}$ 
SCHEME 1: Four-Step Process for the Synthesis of Colloidal-Supported Metal Nanoparticles (CSMNs)

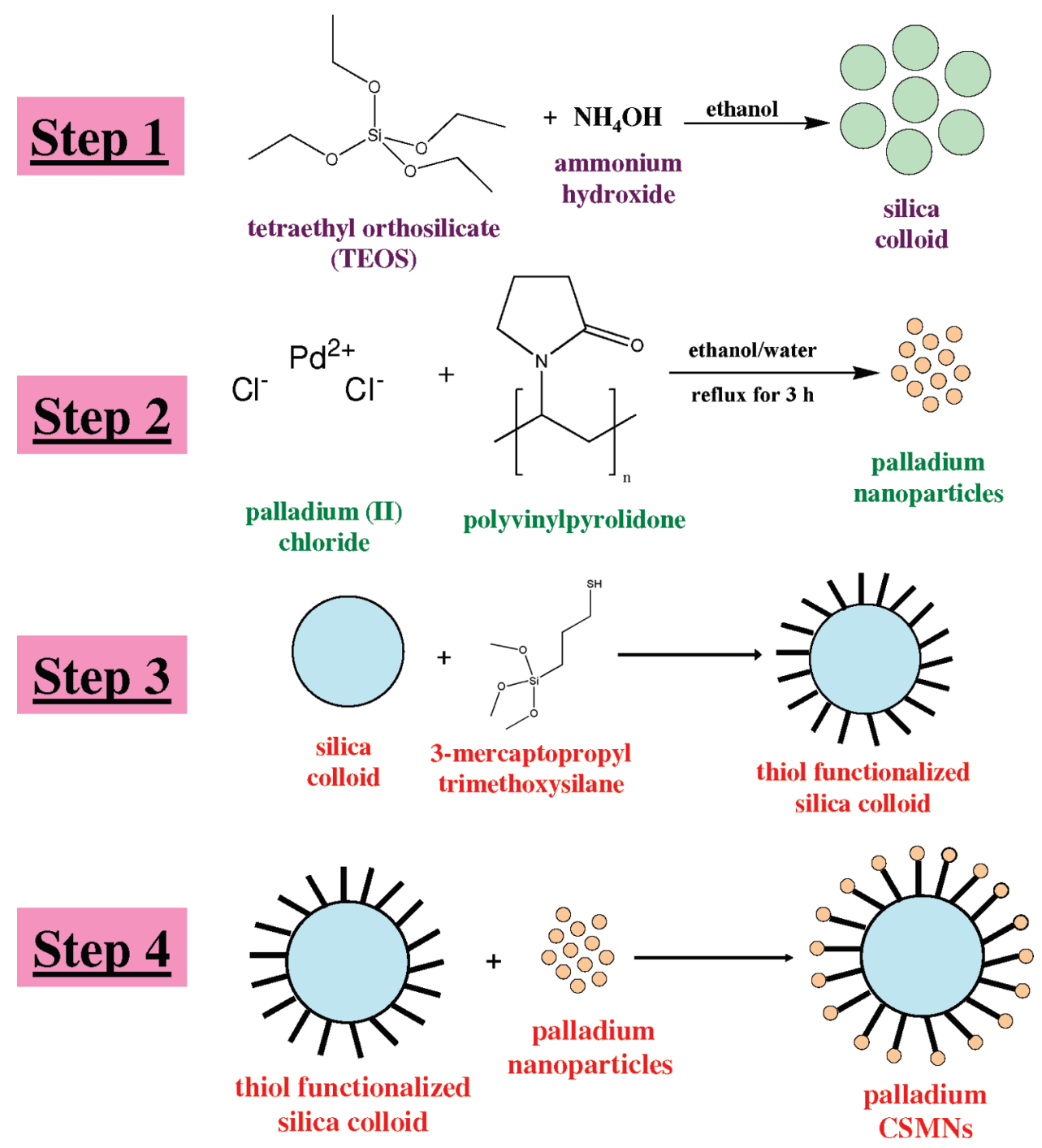

of PVP, and 4 drops of $1 \mathrm{M} \mathrm{HCl}$ was heated. When the solution began to reflux, $14 \mathrm{~mL}$ of ethanol was added. The solution was then refluxed for $3 \mathrm{~h}$, which resulted in a dark brown suspension of Pd nanoparticles.

Functionalization of Silica Colloids. We have used two different linkers to functionalize the silica colloids: 3-mercaptopropyl trimethoxysilane (MPTMS) and 3-aminopropyl triethoxysilane (APTES). Two separate sets of functionalized silica colloids were prepared in which $100 \mu \mathrm{L}$ of MPTMS or APTES is added to the silica colloid suspension.

Synthesis of Colloidal-Supported Palladium Nanoparticles. The functionalized silica colloids were first centrifuged four times at 13,500 rpm for 3 min each time. During the first two centrifugation cycles, the functionalized silica colloids were redispersed in ethanol and during the last two centrifugation cycles, they were redispersed in doubly deionized water. Two milliliters of the centrifuged functionalized silica colloids and $4 \mathrm{~mL}$ of the PVP-capped palladium nanoparticles are placed into a scintillation vial and this suspension is mixed for $24 \mathrm{~h}$ to allow the palladium nanoparticles to bind to the functionalized silica colloids.

Characterization Studies with TEM and EDS. One drop of dilute suspensions of the palladium nanoparticles, silica colloids, and the colloidal-supported palladium nanoparticles was placed on Formvar coated copper grids. The drop was allowed to air-dry for $\sim 1 \mathrm{~h}$. The JEOL 2100EX TEM was used to obtain the TEM images of the palladium nanoparticles, silica colloids, and CSMNs. Energy dispersive spectroscopy (EDS) was used to determine what elements are present in the CSMNs.

Size Distribution Analysis. The UTHSCSA ImageTool for Windows Version 3 image analysis software was used to determine the size distributions of the silica colloids and the palladium nanoparticles. The Distance tool in the Analysis pulldown menu is used to measure the number of pixels in the scale bar of the TEM image. On the basis of the number of pixels for the fixed size associated with the scale bar and measuring the number of pixels for $\sim 200$ nanoparticles in several TEM images, the size of the nanoparticles can be calculated by dividing the number of pixels for the nanoparticles by the number of pixels of the scale bar and multiplying by the fixed size associated with the scale bar of the TEM image. We then plotted the histogram of percent nanoparticles/colloids vs nanoparticle/colloid size and obtained a Gaussian fit to the histogram. From the Gaussian fit, we can determine the average size and standard deviation of the palladium nanoparticles and the silica colloids.

Suzuki Cross-Coupling Reaction. The Suzuki reaction between phenylboronic acid and iodobenzene was catalyzed by using the PVP-Pd nanoparticles as described previously. 25,26,44 For this reaction, $0.49 \mathrm{~g}(6 \mathrm{mmol})$ of sodium acetate, $0.37 \mathrm{~g}$ (3 

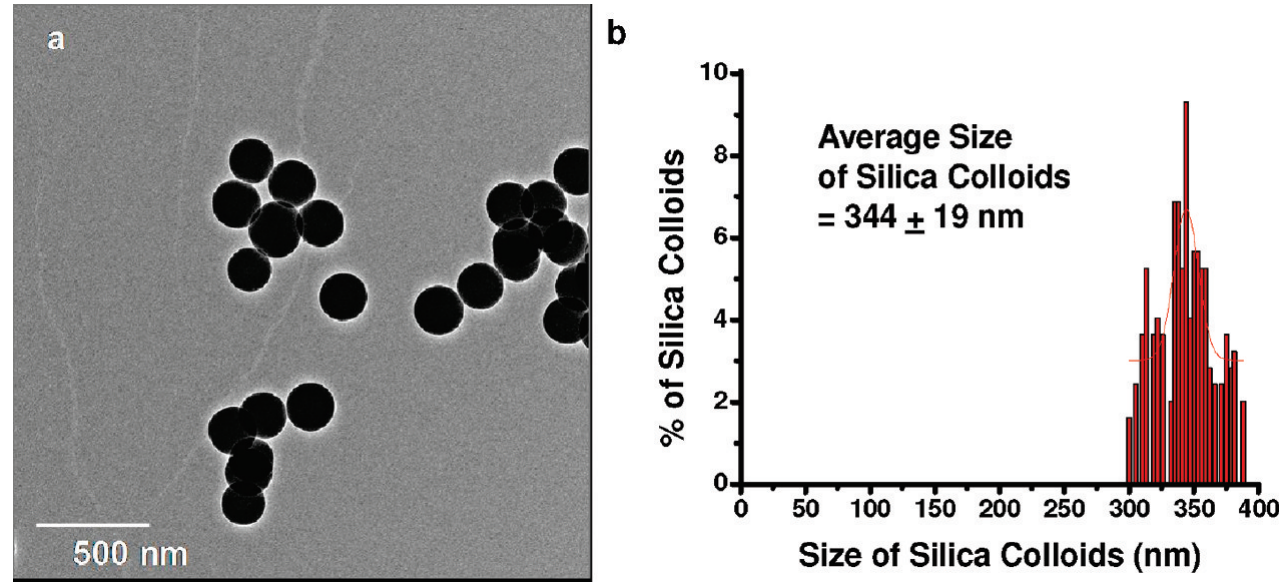

Figure 1. TEM image of the silica colloids synthesized with the Stoeber method (a) and the size distribution histogram (b).
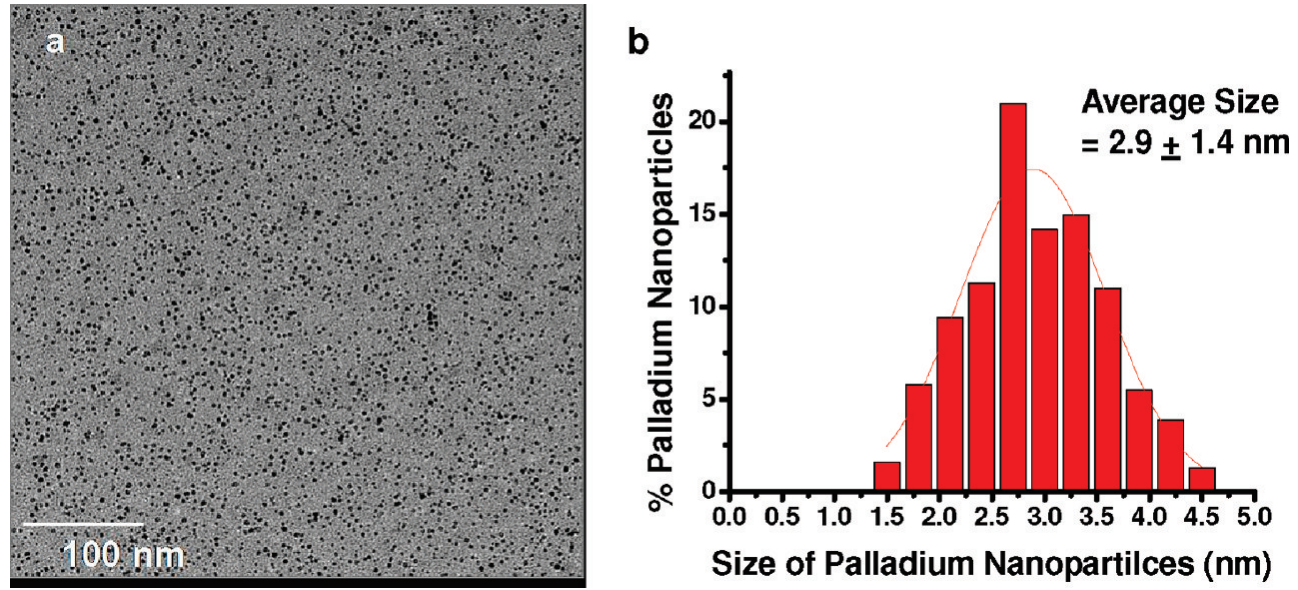

Figure 2. TEM image of the palladium nanoparticles (a) and the size distribution histogram (b).

mmol) of phenylboronic acid, and $0.20 \mathrm{~g}(1 \mathrm{mmol})$ of iodobenzene were added to $150 \mathrm{~mL}$ of 3:1 acetonitrile:water solvent. The solution was heated to $100{ }^{\circ} \mathrm{C}$ and $5 \mathrm{~mL}$ of the PVP-Pd nanoparticles was added to start the reaction. The reaction mixture was refluxed for a total of $12 \mathrm{~h}$.

\section{Results and Discussion}

Synthesis of Colloidal-Supported Metal Nanoparticles (CSMNs). We discuss the design of a new type of intermediate nanocatalyst that we term as colloidal-supported metal nanoparticles (CSMNs). Some important advantages of CSMNs include the following: (1) being suspended in solution during liquid-phase catalytic reactions, (2) having high metal loadings on the silica colloid surface resulting in a high surface area, and (3) facile separation of the reaction mixture from the catalyst. The CSMNs combine the advantages of heterogeneous catalysts in a near-homogeneous format. These advantages make using CSMNs particularly attractive nanocatalysts compared to their colloidal counterparts and those adsorbed onto bulk supports for liquid-phase reactions. Scheme 1 illustrates the fourstep process that we have used to synthesize the palladium nanoparticles supported onto the silica colloids: (1) synthesizing the silica colloids, (2) synthesizing the palladium nanoparticles, (3) functionalizing the silica colloid surface, and (4) attaching the palladium nanoparticles onto the silica colloid surface. The silica colloids were synthesized by using the Stoeber synthesis method and the palladium nanoparticles were synthesized by using the ethanol reduction method and polyvinylpyrolidone as the stabilizer. The silica colloids were functionalized by using two different types of linkers: 3-mercaptopropyl trimethoxysilane (MPTMS) and 3-aminopropyl triethoxysilane (APTES). The palladium nanoparticles were covalently attached to the functionalized silica colloids via the $\mathrm{Pd}-\mathrm{S}$ bond in the case of the silica colloids functionalized with MPTMS and the $\mathrm{Pd}-\mathrm{N}$ bond in the case of the silica colloids functionalized with APTES.

The silica colloids were synthesized by using the Stoeber synthesis method ${ }^{40}$ and TEM was used to characterize the size of the silica colloids. On the basis of the size measurements obtained with ImageTool, we have plotted the size distribution histogram and calculated the average size of the silica colloids. Figure 1 shows a representative TEM image of the silica colloids as well as the size distribution histogram obtained based on the size measurements with the ImageTool image analysis software. The size distribution histogram is plotted as percent silica colloids vs silica colloid size. The average size of the silica colloids is $344 \pm 19 \mathrm{~nm}$ and it can be seen that the silica colloids are relatively monodisperse.

The palladium nanoparticles were synthesized by using the ethanol reduction method as described previously. ${ }^{25}$ Figure 2 shows a representative TEM image of the palladium nanoparticles as well as the size distribution histogram obtained for the palladium nanoparticles. In this case, the size distribution histogram was also plotted as percent palladium nanoparticles vs palladium nanoparticle size. The average size of the palladium nanoparticles is $2.9 \pm 1.4 \mathrm{~nm}$ based on the Gaussian fit of the size distribution histogram. 

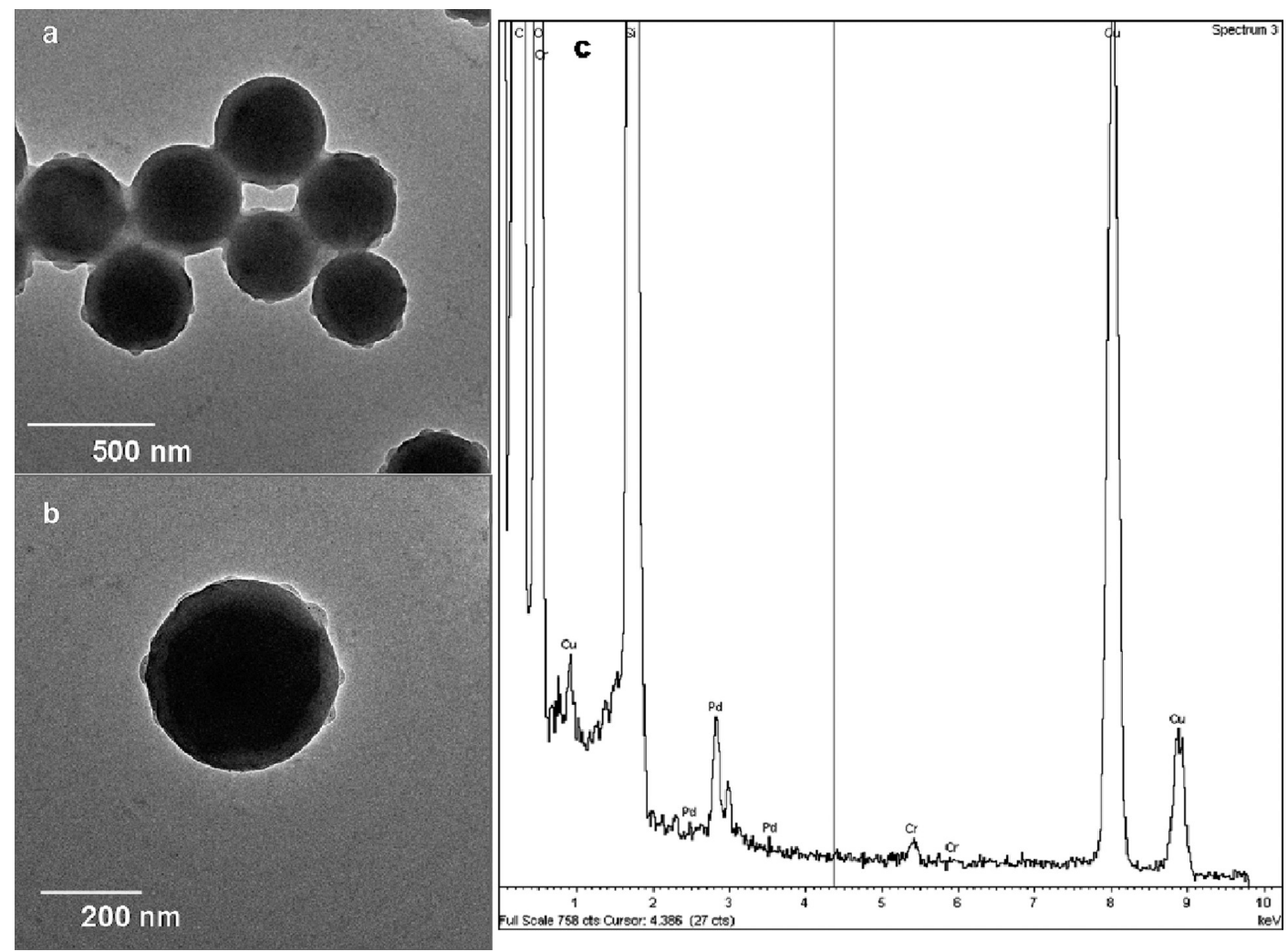

Figure 3. TEM images and EDS spectrum of CSMNs prepared with silica colloids functionalized with 3-mercaptopropyl trimethoxysilane and palladium nanoparticles.

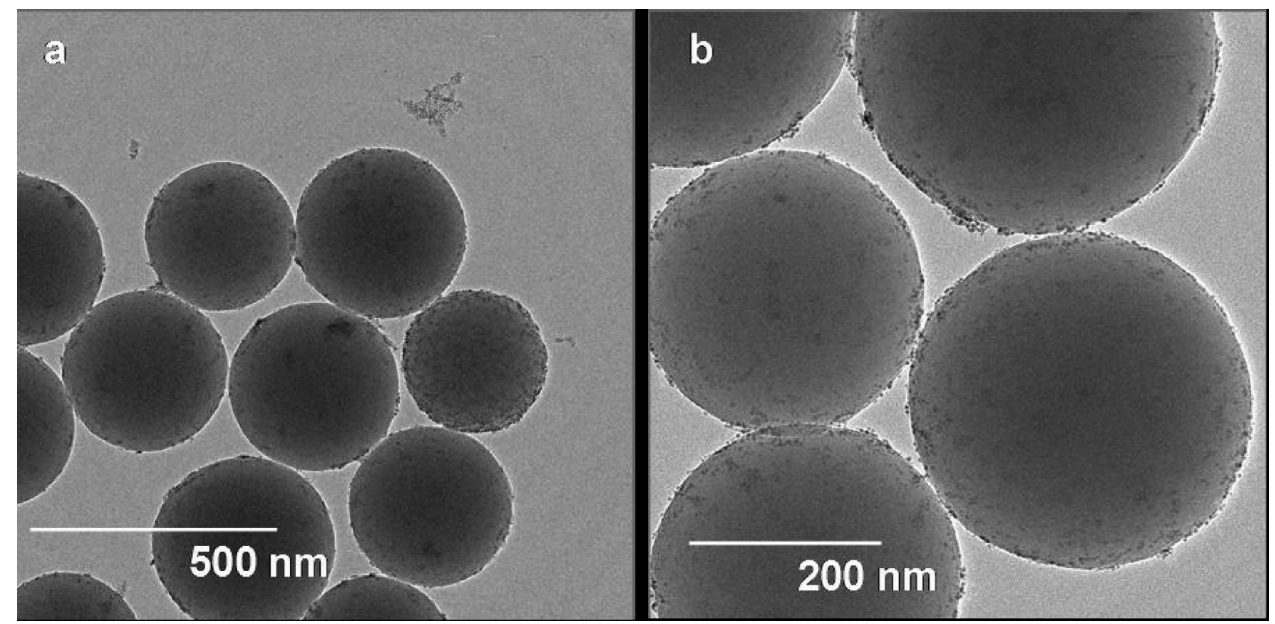

Figure 4. TEM image of CSMNs prepared with APTES-functionalized silica colloids (a) and TEM image of the CSMNs at a higher magnification (b).

Figure 3 shows examples of TEM images of the palladium nanoparticles attached to the silica colloids functionalized with MPTMS. We also obtained the energy dispersive spectrum (EDS) of the CSMNs to determine if both Si and Pd peaks were present. The EDS spectrum can give valuable information on the elements present which can help determine whether we have formed CSMNs. As can be seen in Figure 3, there are peaks associated with both $\mathrm{Pd}$ and $\mathrm{Si}$ that are present, which is evidence for the formation of the CSMNs since this is direct evidence of the attachment of the Pd nanoparticles onto the functionalized silica colloids. The silica colloids functionalized with MPTMS have mercapto groups available for the palladium nanoparticles to bind. This results in the $\mathrm{Pd}-\mathrm{S}$ bond being formed. Also, from Figure 3, it can be seen that there is a significant amount of additional deposits that are present between each of the CSMNs with silica colloids functionalized with MPTMS. We will discuss this finding in more detail later on in another section of this paper.

We have also investigated the attachment of the palladium nanoparticles by using silica colloids functionalized with APTES. We chose this linker since this would result in the silica colloids being functionalized with amine groups that would be available to bind to the palladium nanoparticles. Figure 4 shows examples of TEM images of CSMNs prepared by attaching the palladium nanoparticles to the silica colloids functionalized with APTES. From the TEM images, it can be seen that the palladium nanoparticles readily attach to the Pd nanoparticle surface since in this case the functionalized silica colloids have amine groups available for the Pd NPs to bind. The covalent attachment of the palladium nanoparticles occurs by formation of the $\mathrm{Pd}-\mathrm{N}$ 

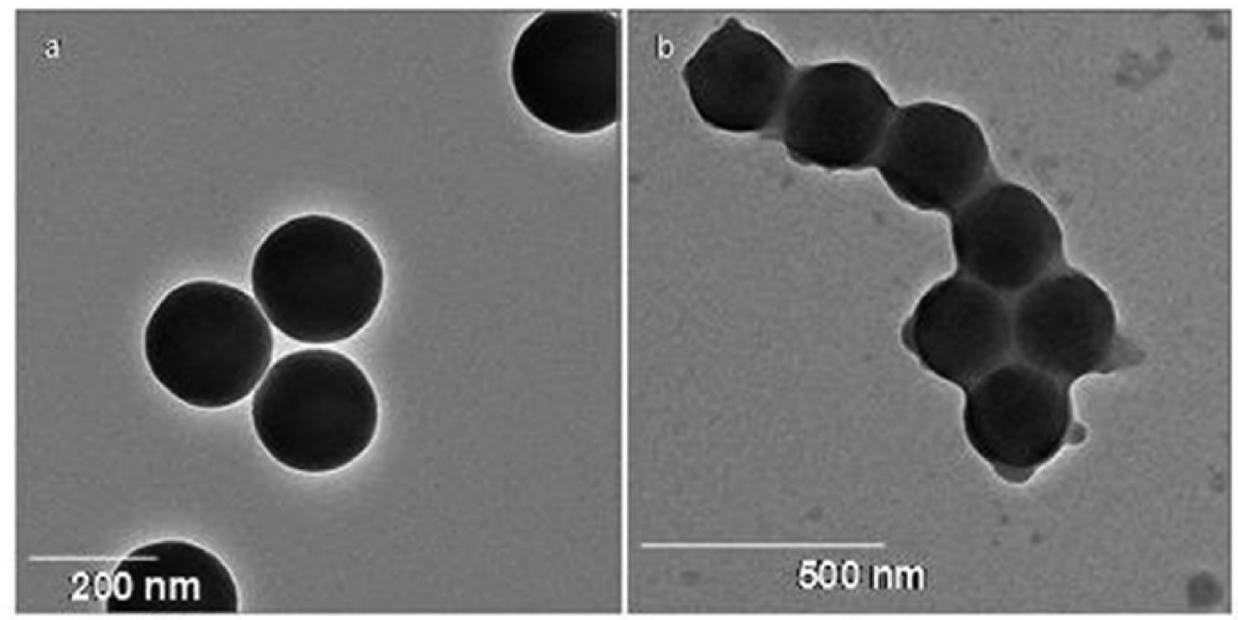

Figure 5. TEM images of the silica colloids before and after functionalization with 3-mercaptopropyl trimethoxysilane.
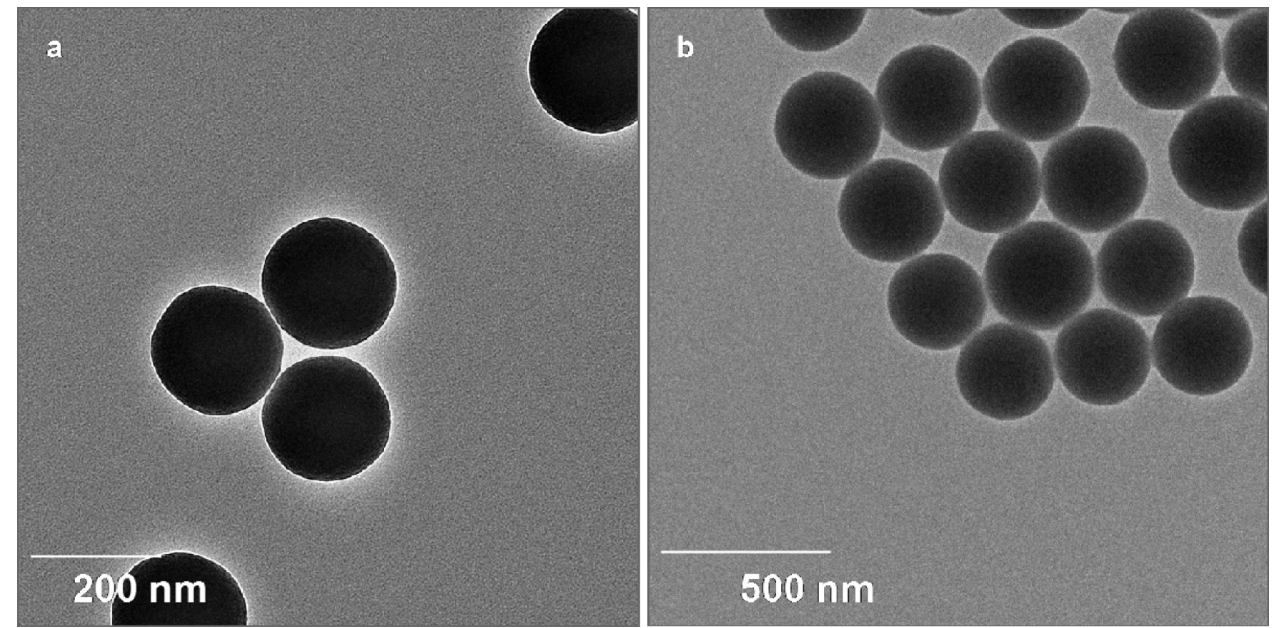

Figure 6. TEM images of the silica colloids before and after functionalization with 3-aminopropyl triethoxysilane.

bond. Also, it can be seen that there is a greatly diminished amount of additional deposits observed for the CSMNs prepared with silica colloids functionalized with APTES. This will be discussed in more detail in the next section of this paper.

Investigations on Additional Deposits Present in CSMNs Prepared with MPTMS-Functionalized Silica Colloids. As we briefly discussed earlier in this paper, we have observed significant additional deposits in the case of the CSMNs prepared with MPTMS-functionalized silica colloids and very little or minimal amounts of these types of deposits in the case of the CSMNs prepared with the APTES-functionalized silica colloids. As a result, we have conducted a set of investigations to determine at what stage the deposits are formed. These studies will help determine whether the deposits occurred after the functionalization process or after the stage in which the palladium nanoparticles were allowed to react with the functionalized silica colloids. To answer this question, we have conducted a set of experiments in which we obtained TEM images of the silica colloids before and after functionalization with MPTMS and APTES.

Figures 5 and 6 show the TEM images of the silica colloids before and after functionalization with MPTMS and APTES. It can be seen that the deposits are formed on the silica colloid surface after functionalizing the silica colloids with MPTMS, but not when the silica colloids are functionalized with APTES. As a result, it can be seen that the formation of the deposits occurs after the functionalization process in the case of the
MPTMS-functionalized silica colloids. This shows that in the case of the CSMNs prepared with silica colloids functionalized with MPTMS, the deposits are formed during the stage in the synthesis where the silica colloids are functionalized with the MPTMS. Also, it can be seen that there are very little or minimal amounts of deposits observed after functionalizing the silica colloids with APTES. This is consistent with the observation that very little or minimal deposits are present in the case of the CSMNs prepared with the silica colloids functionalized with APTES.

This now raises the question of how these deposits are formed and also why there is a significant amount of deposits formed when MPTMS is used as the linker and very little or minimal amounts of deposits formed when the APTES is used as the linker. It is worth noting that that the synthesis of the silica colloids occurs by base-catalyzed hydrolysis of tetraethyl orthosilicate (TEOS). ${ }^{45}$ In the Stoeber synthesis method ${ }^{40}$ to form silica colloids, ammonium hydroxide is used as the base. It is also worth noting that mesoporous silica materials containing mercaptopropyl or phenyl groups on their surface can be prepared by the sol-gel technique. ${ }^{46}$ This technique involves cohydrolysis of TEOS with MPTMS or phenyltriethoxysilane (PTES) in the presence of hexadecyltrimethylammonium bromide as a templating agent in aqueous sodium hydroxide. ${ }^{46}$

This suggests that in our observations of additional deposits being present in MPTMS-functionalized silica colloids, there could be a side reaction that also takes place. After synthesizing 


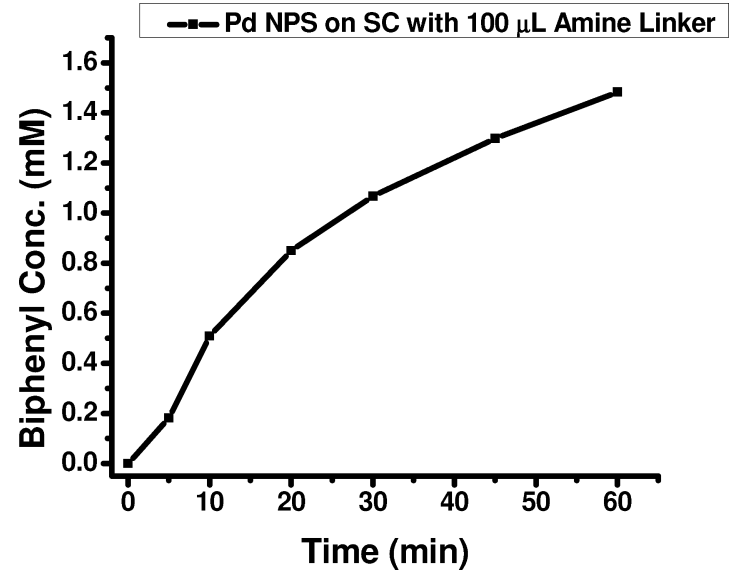

Figure 7. Kinetics of the CSMNs prepared with palladium nanoparticles (NPs) bound to silica colloids (SC) functionalized with the amine linker.

the silica colloids, $100 \mu \mathrm{L}$ of the MPTMS is added directly to the silica colloid suspension. As a result, there could still be some TEOS and ammonium hydroxide also present in the silica colloid suspension. Thus there are two reactions that can occur when the MPTMS is added to the silica colloid suspension. The main and desired reaction involves the functionalization of the MPTMS on the surface of the silica colloids and the mercapto groups being available for the palladium nanoparticles to bind to the MPTMS-functionalized silica colloids. The secondary side reaction involves cohydrolysis of MPTMS with excess unreacted TEOS still remaining in solution in the presence of the ammonium hydroxide base. This secondary reaction would result in the formation of amorphous silica materials and would explain the additional deposits that we observed in the TEM images of both the MPTMS-functionalized silica colloids and the CSMNs prepared by using MPTMS-functionalized silica colloids.

It is also interesting to observe that there are very little or minimal amounts of additional deposits present when the silica colloids are functionalized with APTES and when the palladium nanoparticles are attached to the APTES-functionalized silica colloids. It is quite possible that the cohydrolysis side reaction observed for the MPTMS functionalized silica colloids occurs at a much lower rate for the APTES-functionalized silica colloids. It is worth noting that the cohydrolysis reaction has been reported to occur with many different silanes including MPTMS, APTES, and PTES. ${ }^{46,47}$ One reason that there is a significantly diminished amount of additional deposits present in the APTES-functionalized silica colloids and the CSMNs prepared with APTES-functionalized silica colloids could be that the rate of functionalization onto the silica colloids is much faster than the rate of the cohydrolysis process between TEOS and APTES in the presence of ammonium hydroxide base. In the case of MPTMS-functionalized silica colloids, the functionalization onto the silica colloids occurs at a much slower rate compared to the cohydrolysis process between TEOS and MPTMS in the presence of ammonium hydroxide base. Overall, the secondary cohydrolysis side reaction occurs at a faster rate for the MPTMS-functionalized silica colloids compared to the APTES-functionalized silica colloids. This would also explain why there is a very little or minimal amount of deposits present in the case of the APTMS-functionalized silica colloids and a significantly larger amount of deposits in the case of the MPTMS-functionalized silica colloids. Overall, both types of CSMNS have the potential to be used as intermediate types of nanocatalysts for liquid-phase reactions that require palladiumbased catalysts.

Catalytic Activity and Stability of the CSMNs. We have used the CSMNs prepared with the amine-functionalized silica colloids as catalysts for the Suzuki reaction between phenylboronic acid and iodobenzene to form biphenyl. Reversed phase HPLC was used to follow the kinetics of the biphenyl peak formed during the first hour of the Suzuki reaction between phenylboronic acid and iodobenzene. Figure 7 shows the reaction kinetics during the first hour of the Suzuki reaction. It can be seen that biphenyl starts to form during the first $5 \mathrm{~min}$ of the reaction.

We have also examined the stability of the CSMNs after the full course of the Suzuki reaction (12 h). Figure 8 shows TEM

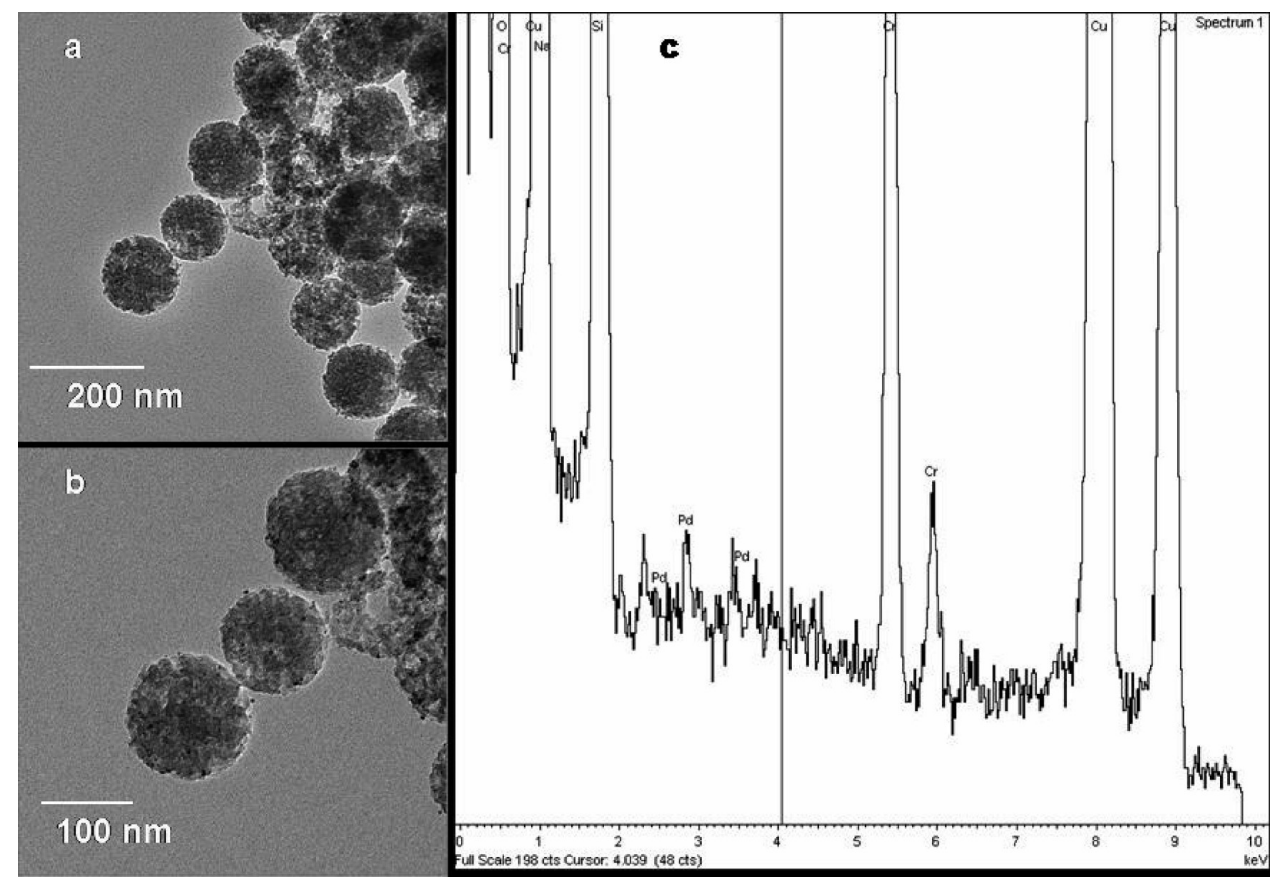

Figure 8. TEM images ( $a, b)$ and EDS spectrum (c) of the CSMNs after the Suzuki reaction. 
images of the CSMNs prepared with the amine-functionalized silica colloids after the Suzuki reaction as well as the EDS spectra. It can be seen that there are structural changes in the silica colloids that take place in which the silica colloids become porous. The observed changes in the silica colloids could be due to the high temperatures of the Suzuki reaction and the long reflux times. The palladium nanoparticles continue to be attached to the silica colloid surface as can be seen in the EDS spectrum in which the Pd peaks are present in addition to the Si peak.

\section{Conclusions}

We have designed a new type of intermediate nanocatalyst that we have termed colloidal-supported metal nanoparticles (CSMNs) that we have characterized by using TEM and EDS. We have prepared the CSMNs by covalently attaching palladium nanoparticles to the MPTMS- or APTES-functionalized silica colloids. The CSMNs would serve as an attractive intermediate nanocatalyst in liquid-phase reactions. In the case of the CSMNs prepared with the MPTMS-functionalized silica colloids, it is observed that there are additional deposits present between the individual MPTMS-functionalized silica colloids. These additional deposits are formed due to a side reaction that involves cohydrolysis of TEOS with MPTMS in the presence of ammonium hydroxide base. The additional deposits are greatly diminished in the case of the APTES-functionalized silica colloids and the CSMNs prepared with the APTES-functionalized silica colloids and this could be due to the cohydrolysis side reaction occurring at a much slower rate. After the full course of the Suzuki reaction, it is observed that there are structural changes in the silica colloids in which it becomes porous and the palladium nanoparticles are still bound to the silica colloids. Overall, both types of CSMNs have the potential to be used as intermediate types of nanocatalysts for liquidphase reactions that require palladium-based catalysts.

Acknowledgment. We thank the University of Rhode Island for the start-up funds that were used to conduct this research. We also thank Dr. Richard Kingsley in the University of Rhode Island Electron Microscopy Center for assistance in acquiring the TEM images and EDS spectra obtained using the JEOL 2100EX transmission electron microscope.

\section{References and Notes}

(1) Narayanan, R.; El-Sayed, M. A. J. Phys. Chem. B 2005, 109, $12663-12676$.

(2) Narayanan, R.; El-Sayed, M. A. Chim. Oggi 2007, 25, 84-86.

(3) Narayanan, R.; El-Sayed, M. A. Top. Catal. 2008, 47, 15-21. $60-74$.

(4) Narayanan, R.; Tabor, C.; El-Sayed, M. A. Top. Catal. 2008, 48 ,

(5) Roucoux, A. Top. Organomet. Chem. 2005, 16, 261-279.

(6) Roucoux, A.; Nowicki, A.; Philippot, K. Nanopart. Catal. 2008, 349-388.

(7) Roucoux, A.; Philippot, K. Handb. Homogeneous Hydrogenation 2007, 1, 217-256.
(8) Wang, D.; Xie, T.; Li, Y. Nano Res. 2009, 2, 30-46.

(9) Thibault-Starzyk, F.; Daturi, M.; Bazin, P.; Marie, O. Nanopart. Catal. 2008, 505-528.

(10) Chan, K.-Y.; Chi, N.; Phillips, D. L. Curr. Top. Electrochem. 1998, 6, 189-197.

(11) Hermans, S.; Khimyak, T.; Raja, R.; Sankar, G.; Johnson, B. F. G.; Thomas, J. M. Stud. Surf. Sci. Catal. 2003, 145, 181-184.

(12) Kumar, R.; Ghosh, A.; Patra, C. R.; Mukherjee, P.; Sastry, M. Nanotechnol. Catal. 2004, 1, 111-136.

(13) Thomas, J. M.; Johnson, B. F. G.; Raja, R.; Sankar, G.; Midgley, P. A. Acc. Chem. Res. 2003, 36, 20-30.

(14) Baeumer, M.; Libuda, J.; Neyman, K. M.; Roesch, N.; Rupprechter, G.; Freund, H.-J. Phys. Chem. Chem. Phys. 2007, 9, 3541-3558.

(15) Somorjai, G. A. Appl. Surf. Sci. 1997, 121/122, 1-19.

(16) Somorjai, G. A. ACS Symp. Ser. 2005, 890, 210-219.

(17) Somorjai, G. A.; Rioux, R. M. Catal. Today 2005, 100, 201-215.

(18) Somorjai, G. A.; York, R. L.; Butcher, D.; Park, J. Y. Phys. Chem. Chem. Phys. 2007, 9, 3500-3513.

(19) Eppler, A.; Rupprechter, G.; Guczi, L.; Somorjai, G. A. J. Phys. Chem. B 1997, 101, 9973-9977.

(20) Burda, C.; Chen, X.; Narayanan, R.; El-Sayed, M. A. Chem. Rev. (Washington, DC, U.S.) 2005, 105, 1025-1102.

(21) Narayanan, R.; El-Sayed, M. A. Nano Lett. 2004, 4, 1343-1348.

(22) Narayanan, R.; El-Sayed, M. A. J. Am. Chem. Soc. 2004, 126, 7194 7195.

(23) Narayanan, R.; El-Sayed, M. A. J. Phys. Chem. B 2004, 108, 57265733.

(24) Antolini, E.; Salgado, J. R. C.; da Silva, R. M.; Gonzalez, E. R. Mater. Chem. Phys. 2007, 101, 395-403.

(25) Narayanan, R.; El-Sayed, M. A. J. Am. Chem. Soc. 2003, 125, 83408347.

(26) Narayanan, R.; El-Sayed, M. A. J. Phys. Chem. B 2004, 108, 8572 8580

(27) Narayanan, R.; El-Sayed, M. A. J. Catal. 2005, 234, 348-355.

(28) Astruc, D.; Lu, F.; Aranzaes, J. R. Ange. Chem., Int. Ed. 2005, 44 , $7852-7872$.

(29) Wang, Q.; Ostafin, A. E. Encycl. Nanosci. Nanotechnol. 2004, 5, 475-503.

(30) Mueller, C.; Nijkamp, M. G.; Vogt, D. Eur. J. Inorg. Chem. 2005, $4011-4021$.

(31) Chan, K.-Y.; Ding, J.; Ren, J.; Cheng, S.; Tsang, K. Y. J. Mater. Chem. 2004, 14, 505-516.

(32) Chan, K.-Y. Small Fuel Cells (5th Ed.) 2004, 305-334.

(33) Chang, J.-S.; Hwang, J.-S.; Park, S.-E. Res. Chem. Intermed. 2003, 29, 921-938.

(34) Chen, C. L.; Mou, C. Y. Nanotechnol. Catal. 2004, 1, 313-327.

(35) Chen, M.; Goodman, D. W. Chem. Soc. Rev. 2008, 37, 1860-1870.

(36) Yadav, G. D. Catal. Surv. Asia 2005, 9, 117-137.

(37) Sun, K.; Liu, J.; Nag, N. K.; Browning, N. D. Recent Res. Dev. Mater. Sci. 2003, 4, 521-540.

(38) Corain, B.; Centomo, P.; Lora, S.; Kralik, M. J. Mol. Catal. A: Chem. 2003, 204-205, 755-762.

(39) Wood, M. A. J. R. Soc., Interface 2007, 4, 1-17. 573 .

(41) Li, Y.; Hong, X. M.; Collard, D. M.; El-Sayed, M. A. Org. Lett. 2000, 2, 2385.

(42) Li, Y.; Boone, E.; El-Sayed, M. A. Langmuir 2002, 18, 4921.

(43) Teranishi, T.; Miyake, M. Chem. Mater. 1998, 10, 594.

(44) Narayanan, R.; El-Sayed, M. A. Langmuir 2005, 21, 2027-2033.

(45) Badley, R. D.; Ford, W. T.; McEnroe, F. J.; Assink, R. A. Langmuir 1990, 6, 792

(46) Rac, B.; Molnar, A.; Forgo, P.; Mohai, M.; Bertoti, I. J. Mol. Catal. A: Chem. 2006, 244, 46-57.

(47) Rahman, I. A.; Jafarzadeh, M.; Sipaut, C. S. Ceram. Interfaces 2008, 35,1883 .

JP100061A 